\title{
Uso dos Medicamentos Genéricos na População do Município de Aurora-CE, no Período de Janeiro de 2018
}

\author{
Luana Fernandes Tavares $^{1}$; Poliana Moreira de Medeiros Carvalho ${ }^{2}$
}

\begin{abstract}
Resumo: A produção de medicamentos genéricos vem crescendo, como também sua utilização, tratando-se de um medicamento que possui o mesmo princípio ativo, composição e ação terapêutica de outra marca que é fabricado por outro laboratório não sendo mais protegido em razão da extinção da patente, tendo o nome de sua substância ativa, sendo utilizado por ser eficaz, seguro e barato. Nesse sentido, o presente estudo teve como objetivo observar a utilização dos medicamentos genéricos em residentes do município de Aurora-CE. Tratou-se de um estudo transversal com a utilização de um questionário estruturado com 100 pessoas residentes na cidade de Aurora-CE com mais de dezoito anos, de ambos os sexos, realizadas as entrevistas nas ruas do centro da cidade, sendo que o instrumento de coleta de dados abrangeu um questionário contendo dados referentes ao uso dos medicamentos genéricos. Quanto à utilização dos medicamentos genéricos em Aurora, foi visto que $58 \%$ dos entrevistados têm o hábito de usar medicamento genérico, $57 \%$ tendo-os em casa, $69 \%$ afirmaram acreditar na sua eficácia e segurança, $79 \%$ aceitando a troca do medicamento de referência pelo genérico realizada pelo farmacêutico e $54 \%$ comprando genérico não apenas devido o preço ser inferior ao de referência, porém $56 \%$ das pessoas não conhecem a diferença entre os medicamentos genéricos, similares e medicamentos de referência, sendo observada a preferência pelos genéricos. Visto que a Política de Medicamentos Genéricos deve divulgar informações sobre o seu uso informando a população, proporcionando aumento do acesso a medicamentos eficazes, seguros e de qualidade, a preços reduzidos.
\end{abstract}

Palavras-Chave: Uso de Fármaco; Terapêutica; Indústria Farmacêutica.

\section{Use of Generic Drugs in the Municipality of Aurora in the Period of January 2018}

\begin{abstract}
The production of generic drugs has been growing, as has its use, being a drug that has the same active principle, composition and therapeutic action of another brand that is manufactured by another laboratory and is no longer protected due to the extinction of the patent, having the name of its active substance, being used for being effective, safe and cheap. In this sense, the present study aimed to observe the use of generic drugs in residents of the municipality of Aurora-CE. This was a cross-sectional study using a structured questionnaire with 100 people living in the city of Aurora-CE over eighteen years old, of both sexes, interviews were conducted on the streets of the city center, and the Data collection included a questionnaire containing data regarding the use of generic drugs. As for the use of generic drugs in Aurora, it was observed that $58 \%$ of the interviewees had a habit of using generic medicine, $57 \%$ had them at home, $69 \%$ said they believed in their efficacy and safety, $79 \%$ generic reference made by the pharmacist and 54\% generic buying not only because the price is lower than the reference, but $56 \%$ of people do not know the difference between generic, similar and reference drugs, with preference being given to generics. Since the Generic Medicines Policy should disclose information about its use informing the population, providing increased access to effective, safe and quality medicines at reduced prices.
\end{abstract}

Keywords: Drug Use; Therapy; Pharmaceutical Industry.

\footnotetext{
${ }^{1}$ Bacharel em Farmácia, Farmacêutica Generalista, Faculdade Santa Maria, Cajazeiras, PB, Brasil. Cursando especialização em Atenção Farmacêutica na Faculdade Juazeiro do Norte - FJN. Contato: luana.tavares353@ gmail.com;

${ }^{2}$ Doutoranda em Ciências da Saúde pela Faculdade de Medicina do ABC Paulista. Cursando Especialização em Gestão e Docência ensino superior Estácio - FMJ. Bacharel em Farmácia, farmacêutica industrial e bioquímica clínica. Mestra em Bioprospecção Molecular pela Universidade Regional do Cariri - URCA. Professora da Faculdade de Juazeiro do Norte- FJN. Professora da Faculdade de Medicina Estácio FMJ, do curso de Farmácia.
} 


\section{Introdução}

As dificuldades encontradas de acesso ao medicamento pela população levaram o Brasil a implantar a política de Medicamentos Genéricos. Os medicamentos genéricos foram efetivamente introduzidos no Brasil, durante o governo de Fernando Henrique Cardoso, pelo então ministro da saúde José Serra através da lei 9.787, de 10 de fevereiro de 1999, autorizando a comercialização de medicamentos com patentes inválidas por qualquer laboratório em embalagem padronizada com uma tarja amarela com um "G" de Genérico (DIAS; LIEBER, 2006).

Um medicamento genérico é um produto que contém uma substância ativa ou fármaco já comercializado, podendo ser na mesma concentração ou dose, destinado às mesmas indicações de outro medicamento registrado e comercializado por outro fabricante, sem, ter identidade de marca, e que surgem após o vencimento do período de proteção patentearia concedida ao produto inovador, sendo designado pela Denominação Comum Brasileira (DCB) ou na sua ausência, pela Denominação Comum Internacional (DCI), e comercializados pelo nome do princípio ativo (STORPIRTIS et al., 2008).

Os medicamentos genéricos podem ser classificados de acordo com diversos parâmetros: podem ser baseados em grupo terapêutico, por grupo anatômico, por predominância de ação nos sistemas: cardiovascular, respiratório, órgão dos sentidos, sistema urinário; além dos medicamentos genéricos classificados como substâncias sujeitas a controle especial, podendo ter importante enfoque a adoção destes medicamentos envolvendo a produção, a comercialização, a prescrição e o uso (PALMERO; SIMÕES, 2006). E como os medicamentos genéricos não precisam realizar investimentos em pesquisa utilizam o desenvolvimento do mercado e divulgação realizados pelo laboratório inovador para comercializarem seus produtos, estes apresentam preços inferiores aos seus similares de marca, sendo a comparação da sua eficácia, segurança e qualidade feita a partir de testes de bioequivalência (STORPIRTIS et al., 2008).

A política de genéricos tem como objetivo promover o uso racional de medicamentos, estimulando a concorrência, onde os consumidores individuais e institucionais terão disponíveis produtos intercambiáveis de diferentes preços, respeitando-se a decisão de não intercambialidade do profissional prescritor. A promoção do uso de medicamentos genéricos deve ser igualmente, objeto de atenção especial, cabendo ao gestor federal identificar mecanismos que favoreçam a consolidação do uso destes produtos. O uso dos medicamentos 
genéricos contribui para o acesso da população a medicamentos de qualidade e de baixo preço, de modo a oferecer benefícios a favor da população, como na prevenção e tratamento de doenças, no qual foram introduzidos no país a fim de promover a redução dos gastos nos serviços de saúde.

O direito do consumidor em relação aos medicamentos genéricos envolve não apenas o direito de conhecer e ter acesso a esses novos produtos, de qualidade garantida e preço mais acessível. Reforça, sobretudo, a necessidade de assegurar que o direito à saúde dos cidadãos não se torne refém de um mercado excludente. Tendo o direito de ter um mercado de medicamentos competitivo, com preços acessíveis e qualidade assegurada. Desta forma, o objetivo do presente trabalho foi observar a utilização dos medicamentos genéricos em residentes do município de Aurora-CE.

\section{Material e Métodos}

O tipo de estudo usado na pesquisa foi quantitativo, adequado para apurar opiniões conscientes dos entrevistados, utilizando instrumentos estruturados como questionários. A pesquisa foi realizada no município de Aurora-CE.

Foi realizado um questionário com pessoas de ambos os sexos, com idade superior a 18 anos e realizada as entrevistas nas ruas do centro da cidade, no mês de janeiro deste ano. Uma amostragem que possibilitou garantir maior precisão dos resultados finais, através de uma amostra coletada com um número de sujeitos igual a 100 (n) habitantes. O tamanho da amostra foi calculado seguindo o método estatístico de Berni, estando à margem de erro para $10 \%$.

As informações foram coletadas por meio de um questionário estruturado (Anexo 1) com perguntas claras e objetivas, já que devem garantir à uniformidade de entendimento das entrevistas e consequentemente a padronização dos resultados.

\section{Características Mercadológicas da Indústria Farmacêutica no Brasil}

As empresas líderes do setor farmacêutico buscam cada vez mais aumentar a sua participação no mercado através de estratégias, como buscar a liderança no mercado por meio de produtos inovadores, gastando em pesquisa e desenvolvimento e em marketing. A competição na indústria farmacêutica é marcada por uma diferenciação de produtos por meio de classes terapêuticas e a integração vertical, formando barreiras à entrada de novos 
competidores no mercado (ROSENBERG; FONSECA; D’AVILA, 1994).

Pode-se considerar importante a integração das políticas voltadas para o desenvolvimento industrial e da inovação, como forma de garantir benefícios econômicos para o país (QUENTAL et al., 2008).

Os medicamentos genéricos podem ser considerados como uma experiência de sucesso na integração das políticas sociais voltadas para um maior acesso da população a medicamentos com qualidade, com as políticas econômicas voltadas para o desenvolvimento industrial, onde a regulação do mercado se dá pela ação de agências reguladoras, que é a ANVISA (Agência Nacional de Vigilância Sanitária) que atua a fim de beneficiar o consumidor por meio do controle sobre os produtos farmacêuticos (QUENTAL et al., 2008).

O sistema de saúde no Brasil é muito complexo, pois engloba tanto os estabelecimentos públicos com unidades de atenção básica, como estabelecimentos privados. No sistema de manutenção da saúde do brasileiro, o estabelecimento de uma Política Nacional de Medicamentos Genéricos foi um passo que teve como objetivo melhorar a qualidade de vida da população brasileira, facilitando o acesso ao medicamento para o tratamento de diversas patologias (GOMES; REIS, 2006).

O crescimento dos gastos em saúde, tanto no setor público quanto no privado acaba levando a busca de métodos alternativos para tornar racionais os gastos e reduzir o consumo de medicamentos (PALMERO; SIMÕES, 2006).

O aumento da expectativa de vida e consequentemente o envelhecimento da população acabam causando maior custo social; mudanças epidemiológicas; novos procedimentos terapêuticos com o uso de medicamentos de alto custo; novas tecnologias, equipamentos e técnicas usadas nos serviços médicos, entre outros fatores, têm contribuído para o aumento dos gastos em saúde, dificultando o financiamento adequado da assistência médica-farmacêutica, afetando diretamente o acesso, a qualidade e o uso racional dos medicamentos (PALMERO; SIMÕES, 2006).

Com a entrada dos medicamentos genéricos no mercado iniciou a concorrência entre os produtos. Isto forçou a baixa de preços dos medicamentos e a população passou a ter acesso a medicamentos, ocorrendo a disputa entre os medicamentos genéricos e os de referência pela conquista do consumidor, dada a intercambialidade do medicamento de referência pelo genérico (PALMERO; SIMÕES, 2006). 


\section{Diferenças entre Medicamentos Genéricos e de Referência}

No Brasil, os gastos das famílias com medicamentos fizeram o governo brasileiro promover uma política de regulamentação de medicamento genérico, possibilitando sua entrada no mercado farmacêutico brasileiro com base na exigência institucional de teste de bioequivalência para a sua comercialização no país, tendo assim, a redução dos custos de tratamentos de algumas doenças (NISHIJIMA, 2008).

O medicamento genérico entra no mercado com um preço inferior ao do seu medicamento de referência, sendo que a credibilidade da substituição (concorrência) de medicamentos estava ligada à reputação dos laboratórios, que acabou se acentuando e afetando de forma positiva a maioria dos preços dos medicamentos pioneiros (NISHIJIMA, 2008).

Os medicamentos genéricos são equivalentes aos medicamentos novos que perderam a patente (medicamento de referência) apresentando a mesma eficácia, a mesma segurança do produto original e intercambialidade por parte do prescritor. Como muitas vezes este profissional é influenciado pela entrada de novos produtos no mercado, com as mesmas propriedades terapêuticas, acompanhadas de grande publicidade sugerindo serem melhores do que os já disponíveis no mercado, a escolha do medicamento acaba sendo ocasionada a partir da avaliação do preço dos medicamentos (RUMEL; NISHIOKA; SANTOS, 2006).

A Investigação e Desenvolvimento de medicamentos originais é um processo muito dispendioso, para que seja possível às empresas rentabilizarem o investimento realizado, estas usufruem de um período de tempo (cerca de 6 ou 10 anos) em que comercializam o medicamento em exclusividade. Após esse período, passa a ser possível a outras empresas fabricar e comercializar medicamentos genéricos os quais, por não precisarem incorporar no preço os custos de Investigação e Desenvolvimento, tem preços significativamente mais baixos levando a produtos de qualidade e baixo custo, beneficiando o consumidor independentemente da sua posição social (GOMES; REIS, 2006).

A concorrência entre os medicamentos genéricos e os respectivos medicamentos de referência se dá quanto aos seus preços. Sendo que os genéricos foram introduzidos com preços menores do que os de referência, apresentando uma significativa diferença que tendeu a aumentar ao longo do tempo, contribuindo para a oferta de medicamentos a preços menores (VIEIRA; ZUCCHI, 2006). 


\section{Testes de Bioequivalência e Biodisponibilidade dos Medicamentos Genéricos}

Os estudos até hoje desenvolvidos na área do medicamento genérico envolvem principalmente a biodisponibilidade dos fármacos, a bioequivalência com o medicamento de referência, como também as dimensões de impacto que esses medicamentos têm causado no mercado farmacêutico. No entanto, sente-se uma lacuna em relação às implicações sociais que o medicamento genérico tem causado na população, principalmente em relação à confiabilidade e aceitabilidade desses produtos pela sociedade (CARVALHO; ACCIOLY JÚNIOR; RAFFIN, 2006).

A bioequivalência assegura que, em nível terapêutico, os medicamentos genéricos são equivalentes aos produtos originais. A biodisponibilidade indica a rapidez e eficácia da atuação do princípio ativo no organismo (MONTEIRO et al., 2005).

A ação terapêutica de um fármaco depende da existência de uma concentração efetiva deste no seu local de ação, durante um período de tempo desejável. Desta forma a disponibilidade do fármaco a partir da forma farmacêutica assume um papel crítico na eficácia clínica de um medicamento, tornando-se por isso necessário caracterizar o desempenho da formulação que o contém como medição adicional de eficácia (STORPIRTIS et al., 2004).

A avaliação da disponibilidade é realizada com base em parâmetros farmacocinéticos calculados a partir dos perfis de concentração plasmática do fármaco ao longo do tempo, apresentando uma fundamental importância, permitindo não só apenas avaliar a qualidade dos medicamentos como também avaliar interações entre fármacos, podendo determinar a influência de vários fatores fisiológicos e patológicos no modo como o fármaco é absorvido pelo organismo (STORPIRTIS et al., 2004).

De acordo com Storpirtis et al. (2004) as preocupações em termos de biodisponibilidade e bioequivalência recaem sobre medicamentos apresentados sob formas farmacêuticas para as quais existem muitos fatores que podem alterar a liberação, dissolução e a absorção do fármaco no organismo. Tais fatores devem ser estudados durante o desenvolvimento farmacotécnico do produto.

\section{Resultados e Discussão}

Na pesquisa sobre a utilização de medicamentos genéricos feita nas ruas do centro da cidade de Aurora - CE verificou-se que dentre as 100 pessoas entrevistadas 66 foram mulheres 
e 34 foram homens entre 18 e 70 anos de idade, podendo-se observar (Tabela 1) que 65\% eram pessoas solteiras, $33 \%$ casadas e $2 \%$ divorciadas.

Tabela 1. Percentual entre o número de pessoas entrevistadas de acordo com a idade, sexo e estado civil do município de Aurora - CE, 2018.

\begin{tabular}{ccc}
\hline Variáveis & N & $\%$ \\
\hline Idade & 69 & 69 \\
Entre 18 - 30 & 18 & 18 \\
Entre 31 - 49 & 13 & 13 \\
Entre 50 - 70 & & \\
Sexo & 66 & 66 \\
Feminino & 34 & 34 \\
Masculino & & 65 \\
Estado civil & 65 & 33 \\
Solteiro (a) & 33 & 2 \\
Casado (a) & 2 & 100 \\
\hline Divorciado (a) & 100 & \\
Total & & \\
\hline
\end{tabular}

Fonte: Elaborada pela autora.

De acordo com estudo transversal desenvolvido na cidade de Ponta Grossa- Paraná, a população alvo de estudo foi constituída de moradores entre 20 e 59 anos de idade completos, realizado o estudo em área de abrangência de uma Unidade Saúde da Família, diferente do estudo realizado nas ruas do centro da cidade de Aurora, na qual tiveram como população alvo de estudo pessoas entre 18 e 70 anos de idade e a prevalência de pessoas entre 18 e 30 anos (Tabela 1) (VOSGERAU; SOUZA; SOARES, 2011).

De acordo com a pesquisa $58 \%$ das pessoas entrevistadas têm o hábito de usar medicamento genérico e $42 \%$ responderam que NÃO (Figura 1). Segundo estudo realizado em Ponta Grossa - Paraná entre os entrevistados que consomem medicamentos, a prevalência de uso de genéricos foi de 14,7\% enquanto que em Aurora a prevalência quanto ao hábito de usar genérico foi de 58\%, mostrando dessa forma confiança no produto (VOSGERAU; SOUZA; SOARES, 2011). 


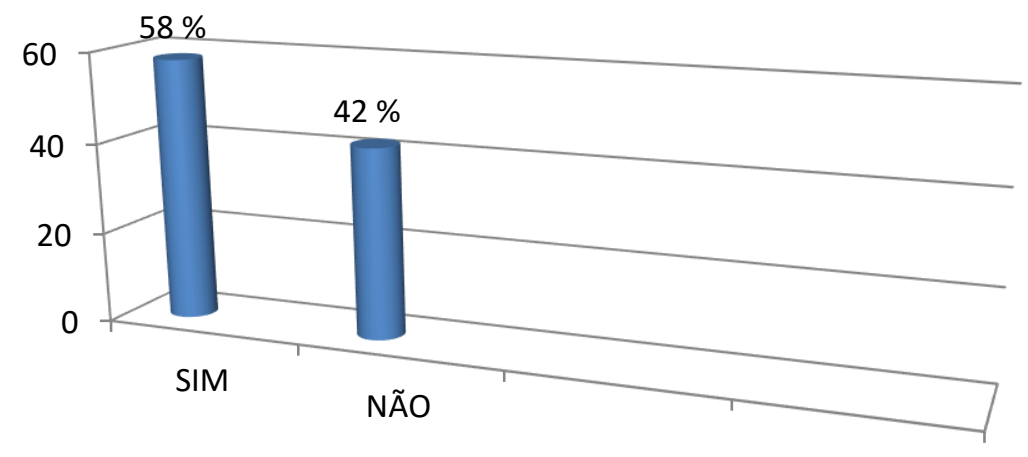

Figura 1. Percentual entre o número de pessoas que demonstraram com o hábito de usar medicamento genérico no município de Aurora-CE, 2018. Fonte: Elaborada pela autora.

As pessoas usam medicamentos genéricos por se tratar de medicamentos que têm a mesma eficácia terapêutica dos medicamentos de marca ou original.

De acordo com Palmero; Simões (2006) em estudo realizado em drogarias na cidade de Rio Claro-SP mostraram que na maioria das drogarias na cidade, a venda de medicamento genérico ainda é baixa, sendo observadas taxas maiores de vendas nas drogarias com tradição de anos no mesmo local, confiança no proprietário ou farmacêutico e oferta de um número maior de medicamentos genéricos. Sendo visto que a utilização de medicamento genérico na cidade de Rio Claro-SP ainda é baixa. Diferentemente da cidade de Aurora o qual foi observado o uso intenso dos genéricos, onde a maioria dos entrevistados disse durante a pesquisa ter inclusive em casa 57\%, medicamentos genéricos (Figura 2).

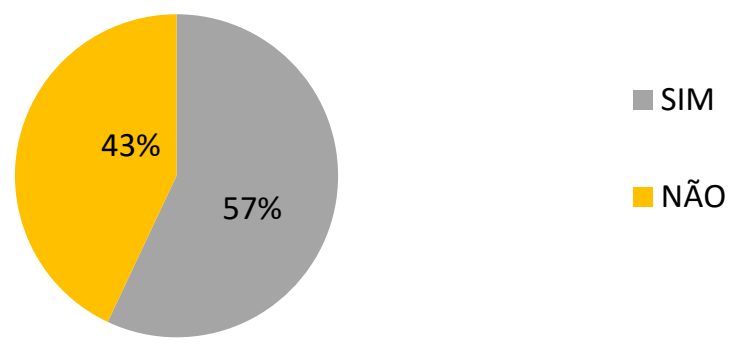

Figura 2. Percentual entre o achado de medicamentos genéricos em casa no município de Aurora - CE, 2018.

Fonte: Elaborada pela autora

De acordo com a pesquisa sobre a utilização de medicamentos genéricos $57 \%$ das pessoas entrevistadas possuem medicamentos genéricos e $43 \%$ disseram não ter. 


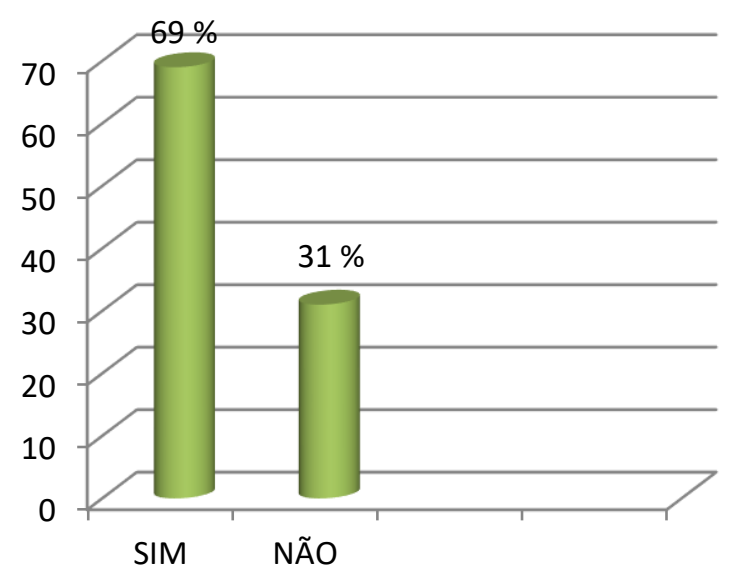

Figura 3. Percentual entre o número de pessoas que dizem acreditar na eficácia e na segurança do medicamento genérico no município de Aurora - CE, 2018.

Fonte: Elaborada pela autora.

Segundo Figueiras; Marcelino; Cortes (2007) a crença na eficácia e segurança dos medicamentos genéricos é forte, sugerindo um grau de confiança considerável neste tipo de fármaco. Sendo que de acordo com o estudo feito com a população portuguesa o que pode explicar a crença na eficácia e segurança dos genéricos são as campanhas promocionais realizadas para promover a credibilidade e a aceitação do medicamento genérico, isto pode explicar confiabilidade observada ao analisarmos os dados, a qual demonstra que $69 \%$ dos entrevistados dizem acreditar na eficácia e segurança dos medicamentos genéricos (Figura 3).

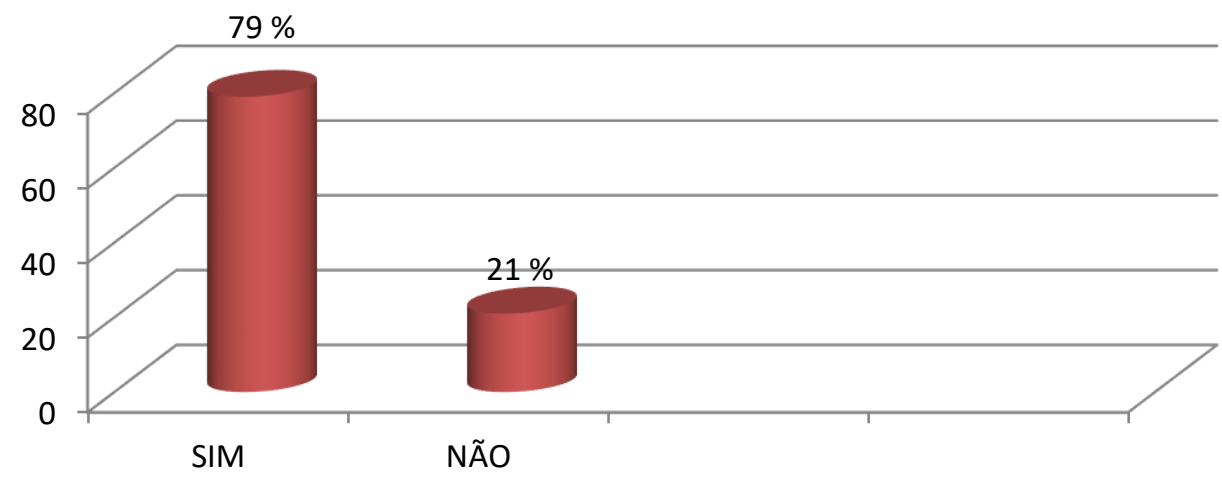

Figura 4. Percentual entre o número de pessoas que dizem aceitar a troca do medicamento de referência pelo medicamento genérico realizada pelo farmacêutico no município de Aurora - CE, 2018.

Fonte: Elaborada pela autora. 
No tocante a intercambialidade dos medicamentos genéricos com os de referência a maioria, $79 \%$, disse estar de acordo com a troca do medicamento inovador pelo genérico feito pelo farmacêutico. Uma vez que o medicamento genérico é intercambiável com o medicamento de referência o paciente tem a chance de procurar o produto mais barato, sem o risco de levar um medicamento com menor eficácia e segurança que a original, no entanto $21 \%$ disseram não aceitar a troca do medicamento de referência pelo medicamento genérico realizada pelo farmacêutico (Figura 4). Este resultado é semelhante a um estudo realizado com usuários de medicamentos genéricos em diferentes drogarias situadas no Distrito Federal - Brasília, onde 90\% dos entrevistados afirmaram aceitar a troca do medicamento de referência pelo medicamento genérico realizada pelo farmacêutico (FERNANDES; COUTINHO; VALLE, 2011).

O preço dos medicamentos sempre foi preocupação constante dos diferentes governos brasileiros por achar que eles são uma barreira ao acesso aos medicamentos. Uma vez que, não se pode descartar que o preço é um dos importantes fatores que dificultam tal acesso, daí a constante preocupação com seu controle e monitoramento.

De acordo com estudo realizado em Ponta Grossa - Paraná foi observado na pesquisa que o uso dos genéricos possivelmente esteja relacionado principalmente devido ao menor custo do medicamento, uma vez que $88,9 \%$ dos entrevistados referiram preços mais baixos para estes produtos farmacêuticos (VOSGERAU; SOUZA; SOARES, 2011), esta situação não foi evidenciada em nosso estudo uma vez que 54\% dos entrevistados afirmaram não comprar o medicamento genérico devido apenas pelo preço ser inferior ao de referência, mas sim pela qualidade que o medicamento genérico apresenta e $46 \%$ das pessoas compram devido o preço ser mais baixo (Figura 5).

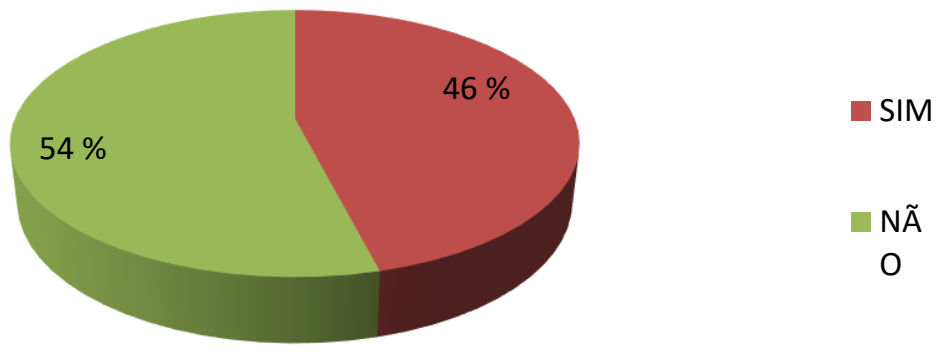

Figura 5. Percentual entre o número de pessoas que dizem comprar medicamento genérico pelo preço ser inferior ao de referência no município de Aurora - CE, 2018.

Fonte: Elaborada pela autora. 
Muitas pessoas ao comprar um medicamento não sabem diferenciar os medicamentos de marca, os similares e os genéricos, a única informação que possuem é que os medicamentos genéricos são mais baratos que os originais e, realmente o são, e ainda possuem a qualidade e eficácia, mas se deve saber que os medicamentos de marca são produtos inovadores, cuja eficácia, segurança e qualidade foram comprovadas cientificamente por ocasião do registro. São medicamentos que estão bastante tempo no mercado e que tem uma marca comercial bastante conhecida. Enquanto que os medicamentos similares são medicamentos que possuem o mesmo fármaco, a mesma concentração, forma farmacêutica, via de administração, posologia e indicação terapêutica do medicamento de referência, mas não tem sua bioequivalência com o medicamento de referência comprovada (STORPIRTIS et al., 2004).

Os medicamentos genéricos são aqueles que contêm o mesmo fármaco (princípio ativo), na mesma dosagem e forma farmacêutica, é administrado pela mesma via e com a mesma indicação terapêutica do medicamento de referência no país, apresentando a mesma segurança, podendo este ser intercambiável. O Ministério da Saúde através da ANVISA avalia os testes de bioequivalência entre o genérico e seu medicamento de referência, apresentados pelos fabricantes, para comprovação da sua qualidade (STORPIRTIS et al., 2008).

Um estudo realizado com pacientes do serviço de saúde ambulatorial do Recife - PE sobre o conhecimento e informação acerca dos medicamentos genéricos, constatou que a falta de conhecimento em relação aos medicamentos genéricos é grande, onde vale salientar que os indivíduos desprovidos de conhecimentos apresentam dificuldades de entendimento quanto à correta e racional utilização dos medicamentos (ROCHA; BARROS; SILVA, 2007). Diferente do estudo em Portugal, onde os resultados mostraram que os indivíduos têm conhecimento satisfatório sobre os genéricos, influenciando assim nas suas decisões em relação ao uso dos medicamentos (FIGUEIRAS; MARCELINO; CORTES, 2007).

Em nosso estudo foi verificado que a maioria das pessoas entrevistadas não conhece a diferença entre os medicamentos de referência, medicamentos genéricos e os medicamentos similares (Figura 6). 


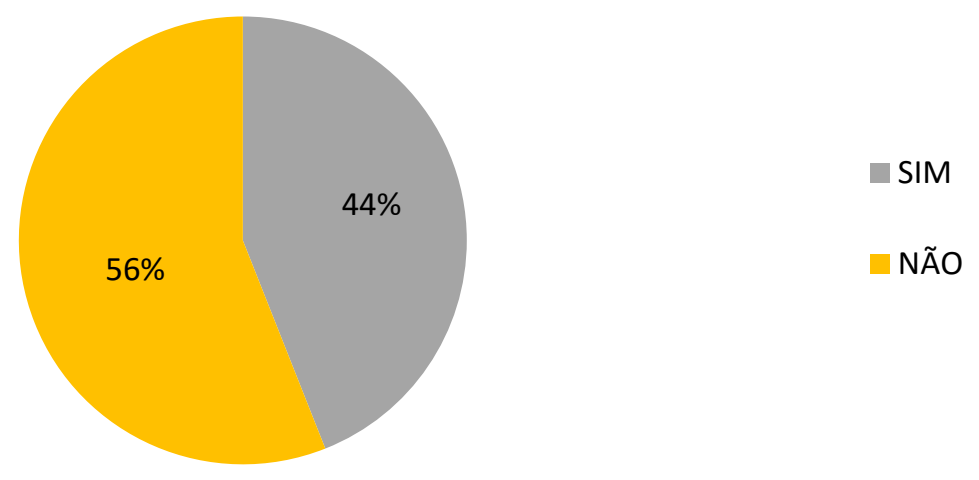

Figura 6. Percentual entre o número de pessoas que dizem conhecer a diferença entre os medicamentos genéricos, similares e medicamentos de referência no município de Aurora - CE, 2018.

Fonte: Elaborada pela autora.

Das pessoas que disseram conhecer a diferença entre os três tipos de medicamentos, a predileção na hora da compra seria pelo medicamento genérico, correspondendo a $61,4 \%$, em segundo lugar o medicamento de referência com $31,8 \%$ e por último compraria o medicamento similar com 6,8\% (Figura 7).

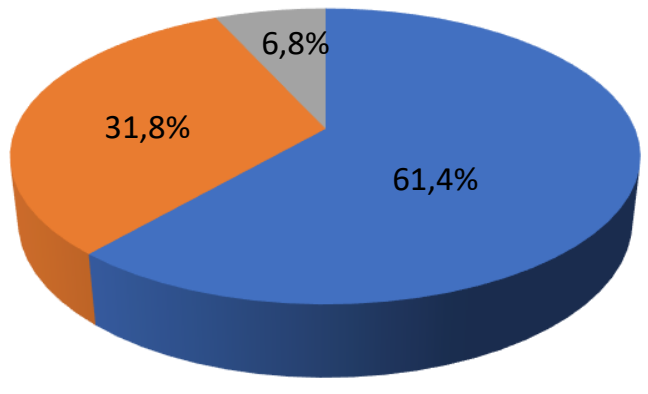

\author{
MEDICAMENTOS \\ GENÉRICOS \\ MEDICAMENTOS DE \\ REFERÊNCIA \\ MEDICAMENTOS \\ SIMILARES
}

Figura 7. Percentual entre o número de pessoas em relação à escolha entre os medicamentos genéricos, similares e medicamentos de referência no município de Aurora - CE, 2018.

Fonte: Elaborada pela autora.

Esta situação está de acordo com estudo realizado em Ponta Grossa - Paraná, onde foi constatado que a maioria $(60,7 \%)$ dos entrevistados desta pesquisa disse que os medicamentos genéricos são a primeira opção na hora da compra (VOSGERAU; SOUZA; SOARES, 2011). Uma pesquisa feita em Viçosa - MG acerca dos genéricos mostrou que o principal motivo para a preferência dos mesmos foi à acessibilidade em termos financeiros, levando assim o 
consumidor a comprar o medicamento (MIRANDA et al., 2010).

\section{Considerações Finais}

A população deste estudo mostrou aceitação pelos medicamentos genéricos, $58 \%$ fazendo uso destes, $69 \%$ acreditando na sua eficácia e segurança, estando satisfeita com os resultados obtidos no emprego; quanto à substituição de medicamentos realizada pelo farmacêutico, a troca do medicamento de referência pelo medicamento genérico é aceita por grande parte dos entrevistados correspondendo a 79\%, manifestando, dessa forma, confiança que parecem ser motivadas muito mais pela experiência de uso do que pelo acesso as informações confiáveis.

A população apresentou dificuldades em diferenciar o medicamento de referência, o genérico e o similar onde correspondeu a $56 \%$; dos que tinham conhecimento sobre os tipos de medicamentos, $61,4 \%$ deu preferência ao genérico por confiar no produto, $54 \%$ deu preferência não apenas devido o preço ser inferior ao de referência.

Medidas são necessárias para que os genéricos tenham cada vez mais sucesso no mercado, e uma importante é a questão da prescrição médica. O produto sendo produzido por indústrias consolidadas reflete em confiança nos profissionais médicos passando a prescrever aos pacientes, que adquire adesão ao novo medicamento.

A política de medicamentos genéricos deve divulgar informações para que a população possa decidir corretamente; com modificações de conceitos principalmente entre os profissionais de saúde, proporcionando então a melhora na qualidade de vida da população.

\section{Referências}

CARVALHO, M. C. R. D. de; ACCIOLY JÚNIOR, H.; RAFFIN, F. N. Representações sociais do medicamento genérico por consumidores residentes em Natal, Rio Grande do Norte, Brasil. Cad. Saúde Pública, Rio de Janeiro, v. 22, n. 3, p. 653-661, mar, 2006.

DIAS, C. R. C.; LIEBER, N. S. R. Processo de implantação da política de medicamentos genéricos no Brasil. Cad. Saúde Pública, Rio de Janeiro, v. 22, n. 8, p. 1661-1669, ago, 2006.

FERNANDES, J. A.; COUTINHO, J. V.; VALLE, M. G. do. Aceitação do medicamento genérico em diferentes níveis de escolaridade e renda familiar do Distrito Federal. Cenarium Pharmacêutico, v. 4, p. 566-576, mai/nov, 2011. 
FIGUEIRAS, M. J.; MARCELINO, D.; CORTES, M. A. Medicamentos genéricos: crenças de senso-comum da população portuguesa. Rev. Port. Clin. Geral, v. 23, p. 43-51, 2007.

GOMES, M. J. V. M.; REIS, A. M. M. Ciências Farmacêuticas: uma abordagem em farmácia hospitalar. 1ed. São Paulo: Atheneu, 2006.

MIRANDA, G. S. et al. Avaliação do conhecimento da população da cidade de Viçosa-MG acerca dos medicamentos genéricos. Rev. Eletrônica de Farmácia, v. 7, n. 3, p. 34-43, 2010.

MONTEIRO, W. M. et al. Avaliação da disponibilidade de medicamentos genéricos em farmácias e drogarias de Maringá (PR) e comparação de seus preços com os de referência e similares. Rev. Brasileira de Ciências Farmacêuticas, São Paulo, v. 41 n. 3, p. 60-69, jul/set, 2005.

NISHIJIMA, M. Os preços dos medicamentos de referência após a entrada dos medicamentos genéricos no mercado farmacêutico brasileiro. RBE, Rio de Janeiro, v. 62, n. 2, p. 189-206, abr/jun, 2008.

PALMERO, E. M. M.; SIMÕES, M. J. S. Estudo comparativo dos medicamentos genéricos com os demais medicamentos em cidade do interior do estado de São Paulo. Rev. Pharmacia Brasileira, Brasília, v. 10, n. 52, p. 8-15, mar/abr, 2006.

QUENTAL, C. et al. Medicamentos genéricos no Brasil: impactos das políticas sobre a indústria nacional. Ciência \& Saúde Coletiva, Rio de Janeiro, v. 13, p. 619-628, 2008.

ROCHA, C. E. da; BARROS, J. A. C. de; SILVA, M. D. P. Levantamento de dados sobre o conhecimento e informação acerca dos medicamentos genéricos em uma população de pacientes do serviço de saúde ambulatorial do Recife, PE, Brasil. Cad. Saúde Pública, Rio de Janeiro, v. 23, n. 5, p. 1141-1150, mai, 2007.

ROSENBERG, G.; FONSECA, M. G. D.; D’AVILA, L. A. Análise comparativa da concentração industrial e de turnover da indústria farmacêutica no Brasil para os segmentos de medicamentos de marca e genéricos. Economia e Sociedade, Campinas, v. 19, n. 38, p. 368-378, jul/set, 1994.

RUMEL, D.; NISHIOKA, S. de A.; SANTOS, A. A. M. Intercambialidade de medicamentos: abordagem clínica e o ponto de vista do consumidor. Rev. Saúde Pública, Rio de Janeiro, v. 40, n. 5, p. 921-7, 2006.

STORPIRTIS, S. et al. A Equivalência Farmacêutica no contexto da intercambialidade entre medicamentos genéricos e de referência: bases técnicas e científicas. Infarma, São Paulo, v. 16, n. 9, p. 51-58, 2004.

STORPIRTIS, S. et al. Farmácia Clínica e Atenção Farmacêutica. Rio de Janeiro: Guanabara Koogan, 2008.

VIEIRA, F. S.; ZUCCHI, P. Diferenças de preços entre medicamentos genéricos e de referência no Brasil. Rev. Saúde Pública, Rio de Janeiro, v. 40, n. 3, p. 444-9, 2006.

VOSGERAU, M. Z. S.; SOUZA, R. K. T. de; SOARES, D. A. Utilização de genéricos em área de atuação da equipe de Saúde da Família em município do sul do Brasil. Rev. Bras. Epidemiol, v. 14, n. 2, p. 253-263, 2011.

\section{Como citar este artigo (Formato ABNT):}

TAVARES, Luana Fernandes; CARVALHO, Poliana Moreira de Medeiros. Uso dos Medicamentos Genéricos na População do Município de Aurora-CE, no Período de Janeiro de 2018. Id on Line Rev.Mult. Psic., 2018, vol.12, n.42, Supl. 1, p. 662-675. ISSN: 1981-1179.

Recebido: $14 / 11 / 2018$

Aceito: $16 / 11 / 2018$ 


\section{ANEXO 1}

Instruções: A seguir são apresentadas questões em que você deverá responder de maneira mais sincera possível, sem deixar nenhuma em branco; não existem respostas certas ou erradas, apenas solicitamos que responda.

\section{Questionário}

1. Idade: anos

2. Estado Civil:

( ) casado ( ) solteiro ( ) divorciado ( ) viúvo

3. Sexo:

( ) masculino ( ) feminino

4. Você tem o hábito de usar medicamento genérico?

( ) $\operatorname{sim}($ ) não

5. Você tem no momento algum medicamento genérico em casa?

( ) $\operatorname{sim}(\quad)$ não

6. Você acredita na eficácia e segurança do medicamento genérico?

( ) $\operatorname{sim}(\quad)$ não

7. Você aceita a troca do medicamento de referência pelo genérico realizada pelo farmacêutico?

( ) $\operatorname{sim}($ ) não

8. Você compra medicamento genérico devido o preço ser inferior ao de referência?

( ) $\operatorname{sim}($ ) não

9. Além dos medicamentos genéricos e de referência existe os medicamentos similares. Você conhece a diferença entre estes medicamentos?

( ) $\operatorname{sim}($ ) não

10. Se você conhece a diferença, diante dos 3 tipos de medicamentos encontrados na farmácia, qual você compraria?

Medicamento de referência ( )

Medicamento similar ( )

Medicamento genérico ( ) 